\title{
Microstrip Antenna 2.4 Ghz U-Slolt Patch Dual Slit Vertical with Ground Square Design for Zigbee Technology
}

\author{
Sri Marini ${ }^{1{ }^{*}}$ Sukwati Dewi Asrika ${ }^{1}$ Andi Hasad ${ }^{1}$ Muhammad Fikri Bivani $^{2}$ \\ Muhammad Viki Nisfani ${ }^{2}$
}

\author{
${ }^{1}$ Islam 45 Bekasi University, 17113, Indonesia \\ ${ }^{2}$ Singa Perbangsa Karawang University, 41361, Indonesia \\ *Corresponding author. Email: srimarini@unismabekasi.ac.id
}

\begin{abstract}
In recent years, ZigBee technology has received many significant developments and high attention as the license free ISM frequency spectrum is available for commercial communications and applications. ZigBee's attractive nature coupled with rapid growth in wireless communication systems has turned ZigBee into an excellent substitute for today's popular and traditional wireless technology, such as Bluetooth. Dual slit vertical U-Slot Patch with ground square design Microstrip Antenna for $2.4 \mathrm{GHZ}$ band applications is presented in this paper. ZigBee's potential is huge because of its enormous advantages, such as the ability to deliver extremely fast data rates at short transmission distances and requiring very low power dissipation. Printed antennas recently played a major role in the development of various frequencies of antennas. The proposed antenna is made of a $1.6 \mathrm{~mm} \mathrm{FR}-4$ epoxy substratum and $\square \mathrm{r}=4.4$. ground level technique with proximity coupling, the antenna proposed works $2.4 \mathrm{GHZ}$ with Vertical patterns of radiation. Its bandwidth of narrow band impedance protects it against interference from other ISM band applications. The proposed antenna is (1.8x1.8) $\mathrm{mm}^{2}$ compact in size. The conductor material uses $0,035 \mathrm{~mm}$ thick copper with simple design, narrow bandwidth, compact and easy to manufacture advantages. The results measured are well-conformed with the simulated frequency of $2,4 \mathrm{GHz}$ and vertical radiation model, gain of $\geq 3,5 \mathrm{dBi}$, input impedance $50 \Omega$, returnloss of $\leq-10 \mathrm{~dB}$ and VSWR of $\leq 1,7$.
\end{abstract}

Keywords: Microstrip Antenna, Zigbee, WPAN, U-Slot, Dual Slit Vertikal.

\section{INTRODUCTION}

WPAN is a wireless network that can connect one device to other adjacent devices using interfaces such as Bluetooth, UWB (Ultra-Wideband) and ZigBee. The characteristics of the WPAN have a range that is not so wide (short range) but has the advantages of low power consumption (low power), low cost (low cost), one network can support a minimum of 16 devices [1]

The wireless networking standard ZigBee fits into a market that simply does not come with other wireless technologies. While most wireless standards strive to accelerate, ZigBee seeks to achieve low data rates. While other wireless protocols add more functionality, ZigBee aims for an eight-bit microcontroller-fitting small pile. While other wireless technology seeks to provide the Internet with the last mile of streaming or high-definition media, ZigBee tries to control a light or send temperature information to a thermostat. While other wireless technologies are designed to run on batteries for hours or perhaps days, ZigBee has been developed for years [2].

The wireless control market has several unique needs, for which ZigBee is perfectly suited, as ZigBee is very reliable, cost-effective, very powerful, highly safe and open world standard. ZigBee added a constraint to the technology to achieve the low power and low-cost criteria: Low rate of data Antenna is the main wireless system component. An antenna must have a bandwidth more than $0.25 \mathrm{GHz}$ so it can operate at $2.4 \mathrm{GHz}$ frequency center. The way to obtain a large bandwidth and high gain is on the feeding technique. $2.4 \mathrm{GHz}$ frequency can be used for Wireless Local Area Network (WLAN) and WPAN within data rate around $0.25 \mathrm{Gbps}$ that can enable to transfer HD video signal from a video player to a television [2]. ZigBee is about to become the global network control/sensor standard. It was designed 
to provide the following characteristics: - Simply implemented, low power consumption [3]. To create a wireless network that sends information, data rate, coverage zone, network topology, network size, protocol routing and consumption power can be approximated. WLAN (Wireless Local Area Network) and WPAN divide into two groups for solving this problem (Wireless Personal Area Network). WPAN is a new wireless system that has low data rate, low power consumption and good coverage. Zigbee is a type of WPAN group that uses sensors and home controls [4].

However, the use of a microstrip antenna is a major disadvantage of a small impedance bandwidth. Different techniques to overcome this problem can be found in literature. In [5], the authors used the CPW feeding technique to improve the patch antenna's impedance bandwidth. X.L. Sun and others [6]. The proposal included a patch antenna structure with compact radiator patches covered with improved impedance bandwidth characteristics for all WLAN bands, i.e., 2.4/5.2/5.8 GHz. Meander lines [7] and miniaturized antennas associated with PIFA [8] are also used in the literature for the same purpose. The introduction of Uslots on the patch antenna is however a very popular way of achieving broadband characteristics [9]. The narrow bandwidth and low gain of the version of microwave antennas are so simple and efficient method of achieving the desired compactness, multi-band, and broadband features by loading U slot with recto radiation patch [10].

ZigBee uses IEEE 802.15.4 in full and operates world-wide on the following frequencies in unlicensed bands: $2.400-2.484 \mathrm{GHz}, \quad 902-928 \mathrm{MHz}$ and $868.0-$ $868.6 \mathrm{MHz}$. The ZigBee protocol has all the advantages of the 802.15.4 protocol with additional networking features [9]. An inset feed is a typical way to achieve such an antenna. The inserted feeder distance of $1 \mathrm{~mm}$ can be set to match the feeding edge of the antenna to the characteristic impedance of the transmission line [11].

\section{METHOD}

Designing an antenna microstrip using the help of a software Ansoft HFSS v.13, it will need a validation for proving that the research in the simulation using a software has meet the standard procedure for an antenna microstrip design.

Depend on the reference paper. the writer make the design with basic specification design from the reference paper. First, the writer re-design the antenna using software, then input all the geometry and substrate. Next input the parameter in the software, after the simulation process finish the frequency band obtained and then the writer do some trial with adjusting some parameter and antenna geometry like the number of the slot, the number of the patch and the stub position.

\subsection{Radiation Mechanism and Antenna Structure}

By solving Maxwell's equations in a given antenna structure the radiation pattern and the input impedance are determined. Depending on the solution technique adopted, the problem is either selected to prescribe an integral or differential form of Maxwell's frequency domain equations or the time domain. A combination of forms or domain equations is sometimes used to describe complex antenna problems. These equations are solved using various numerical methods, like Maxwell's integral form, which are solved using the moments method (MoM) [12].

\subsection{Feeding Techniques}

A feedline is used for direct or indirect contact radiation. There are many different feeding techniques and four most popular techniques are coaxial sensor feeding, microstrip, opening connecting and proximity connecting [13]. The coaxial probe feeding system is a feeding method in which the coaxial internal conductor is attached to the antenna radiation patch while the external conductor is connected to the ground plane. Coaxial feeding benefits are easy to manufacture, easy to match and low spurious radiation.

\subsection{Aperture Coupled Feed Patch Antenna}

The opening coupling feed consists of two distinct substrates separated by a ground plane. There is a microstrip feed line on the bottom side of the lower substrate, which is coupled by the patch through a slot on the ground floor separating two substrates. This system allows the feed mechanism and the radiating element to be optimized independently. Usually, the top substratum is a thick, low dielectric constant substratum whereas it is the high dielectric substratum for the bottom substratum. The ground plane in the middle isolates the radiation element's feed and minimises spurious radiation interference for pattern formation and pure polarization. The advantages are that the feed mechanism element can be independently optimized.

\section{RESULT AND DISCUSSION}

\subsection{The Simulation of the Antenna}

This antenna design using Ansoft HFSS (High Frequency Structure Simulation) v.13 for designing and for running the simulation of the antenna. Figure 1 showed the overall design of the antenna every layer 


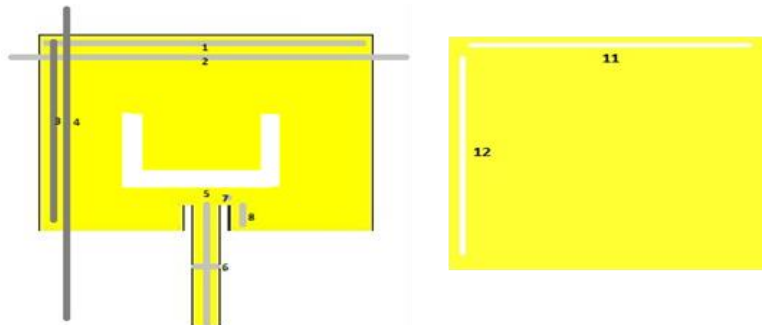

Figure 1 Antenna microstrip design

The Geometric of Upper Layer Substrate Antenna:

1. Patch length

2. Substrate length

3. patch width

4. Substrate length

5. Feeder length

6. Feeder width

7. Slit length

8. Slit width

9. Substrate Thick

10. Patch Thick

The Geometric of Ground Layer Substrate:

11. Ground length

12. Ground width

The geometry from overall antenna design that is Patch (Upper layer width) $=18 \mathrm{~mm}, \mathrm{~L}$ (substrate length) $=18 \mathrm{~mm}$, The primary design parameters are listed Table 1 .

Table 1. Initial Design Parameters.

\begin{tabular}{lll}
\hline & Substrate & Size $(\mathrm{mm})$ \\
\hline 1 & Patch length & 18 \\
2 & Patch width & 18 \\
3 & Dielectric Constant $(\varepsilon r)$ & 1.6 \\
& Ground Planar & $1.8 \times 1.8$ \\
4 & Conductor thickness & 0.035 \\
5 & Partitivities relative & 4.4 \\
6 & Slit width & 1 \\
7 & Slot width & 1 \\
\hline
\end{tabular}

\subsection{Antenna Prototype}

Figure 2 showed the geometric of upper layer substrate with a rectangle patch in the center of the substrate.

The geometry of patch is $L$ (patch length) $=18 \mathrm{~mm}$ and $W($ patch width $)=18 \mathrm{~mm}$. The antenna specifications used can be seen in table 1 . The working frequency used is $2.4 \mathrm{GHz}$. Unlike the previous research which was at a frequency of $915 \mathrm{MHz}$ and $1.2 \mathrm{GHz}$ and adding double Vertical with a gap of $1 \mathrm{~mm}$ in the effective patch resulted in better parameter values.

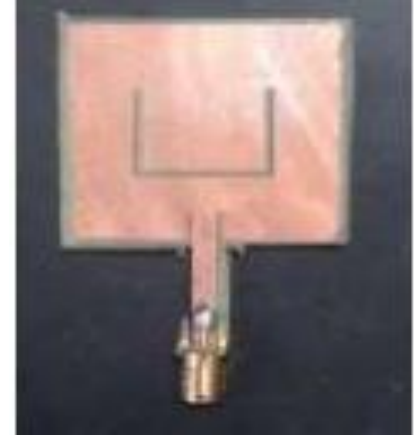

Figure 2 Antenna prototype

ZigBee takes full advantage of the physical radio specification of IEEE 802.15.4 and operates worldwide on the following frequencies in unlicensed bands: 2,400$2,484 \mathrm{GHz}, 868,0-868,6 \mathrm{MHz}$ and $902-928 \mathrm{MHz}$. The ZigBee protocol has all the advantages of the 802.15.4 protocol with an additional networking feature. The ZigBee protocol was designed by the ZIGBEE Alliance, a non-profit consortium of leading semiconductor producers, technology providers, OEMs and end-users worldwide. The resulting parameter values correspond to the specifications of the ZigBee antenna.

\subsection{Implementation Antenna Zigbee Technology to STB}

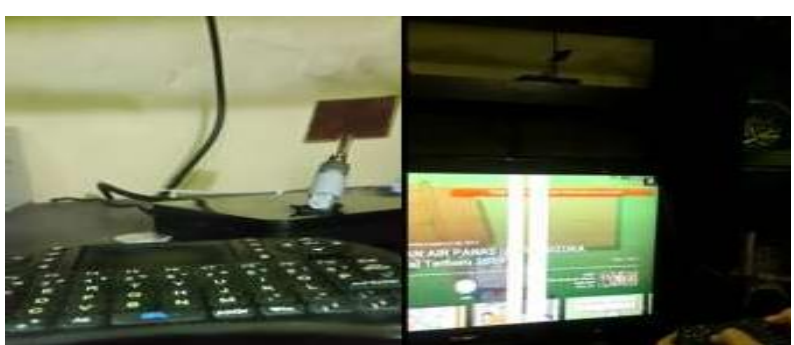

Figure 3 Antenna Zigbee Technology to STB

The Zigbee antenna is installed on the STB and serves the WPAN network to connect to the internet and presented at Figure 3. In testing the antenna by opening the youtube video, the STB is controlled using a remote based on a wireless sensor network. The bandwidth used is from $98 \mathrm{kbps}$ to $5.27 \mathrm{mbps}$ with an average traffic of $6.77 /$ hour and a data rate of $244286 \mathrm{mb}$ with a frequency of $2.4 \mathrm{GHz}$, resulting in video without buffering and a minimum of signal interference showing that the antenna can work with ZigBee technology. Figure 4 shows the average traffic data rate antenna. 

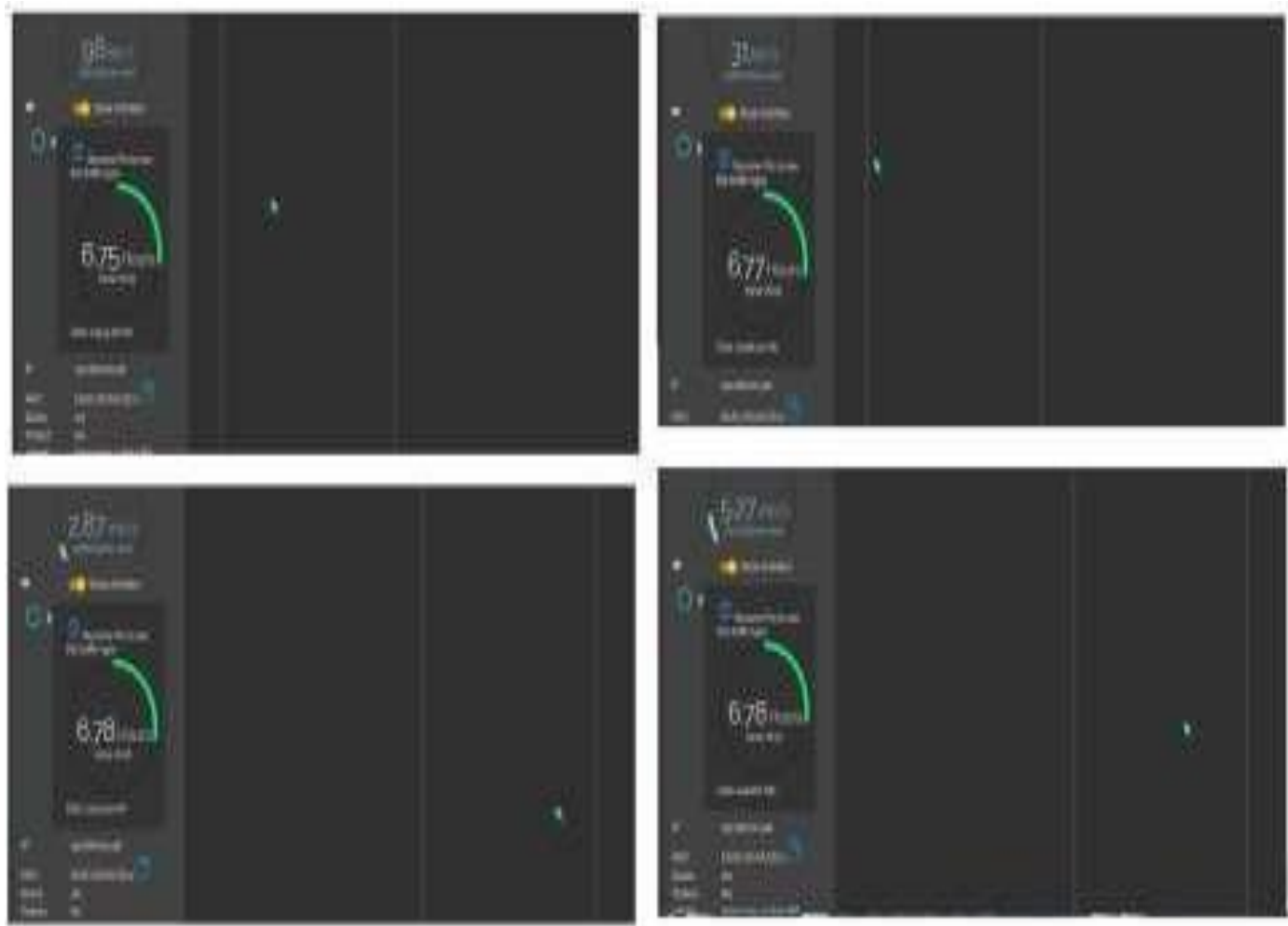

Figure 4 Average traffic data rate antenna

The result of the microstrip antenna Patch Dual Slit Vertical with Ground Square Design for Zigbee Technology using Ansoft HFSS (High Frequency Structure Simulation) v.13 software will be explained in this session. Several parameters will be measured in this session:
a. Bandwidth
b. Return loss
c. Voltage Standing Wave Ratio (VSWR)
d. Gain

The explanation of the measured parameter is explained below:

\subsubsection{Bandwidth And Return Loss}

Bandwidth that has been obtained from the software simulation is $2.4 \mathrm{GHz}$. From the simulation result that has been done, the bandwidth result has been obtain has meet the criteria that is more than $0.25 \mathrm{GHz}$. Figure 5 shows the Return loss curve with optimized parameters.

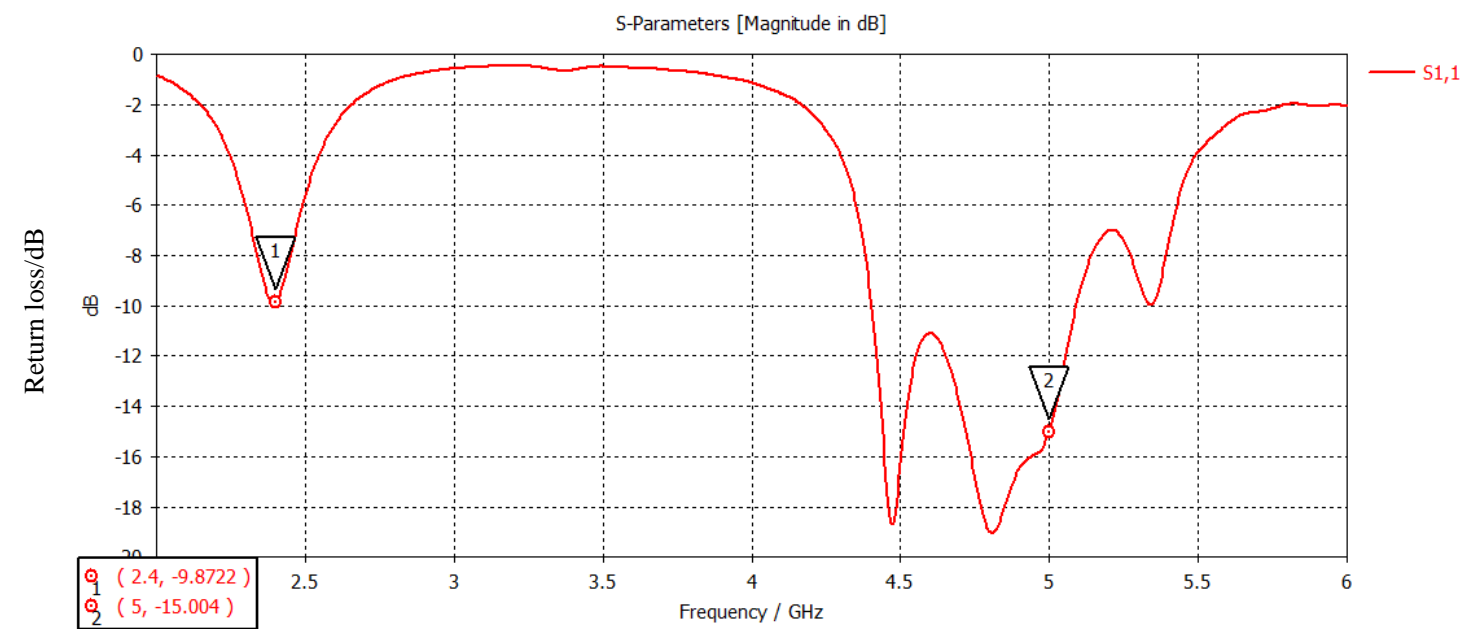

Figure 5 Return loss curve with optimized parameters. 


\subsubsection{Voltage Standing Wave Ratio (VSWR)}

Depending on the measurement result on HFSS that has been done at the frequency from $2.4 \mathrm{GHz}$ until 2.425
$\mathrm{GHz}$ has been obtained a VSWR value of 1.7. The VSWR graph from the simulation can be seen in Figure 6.

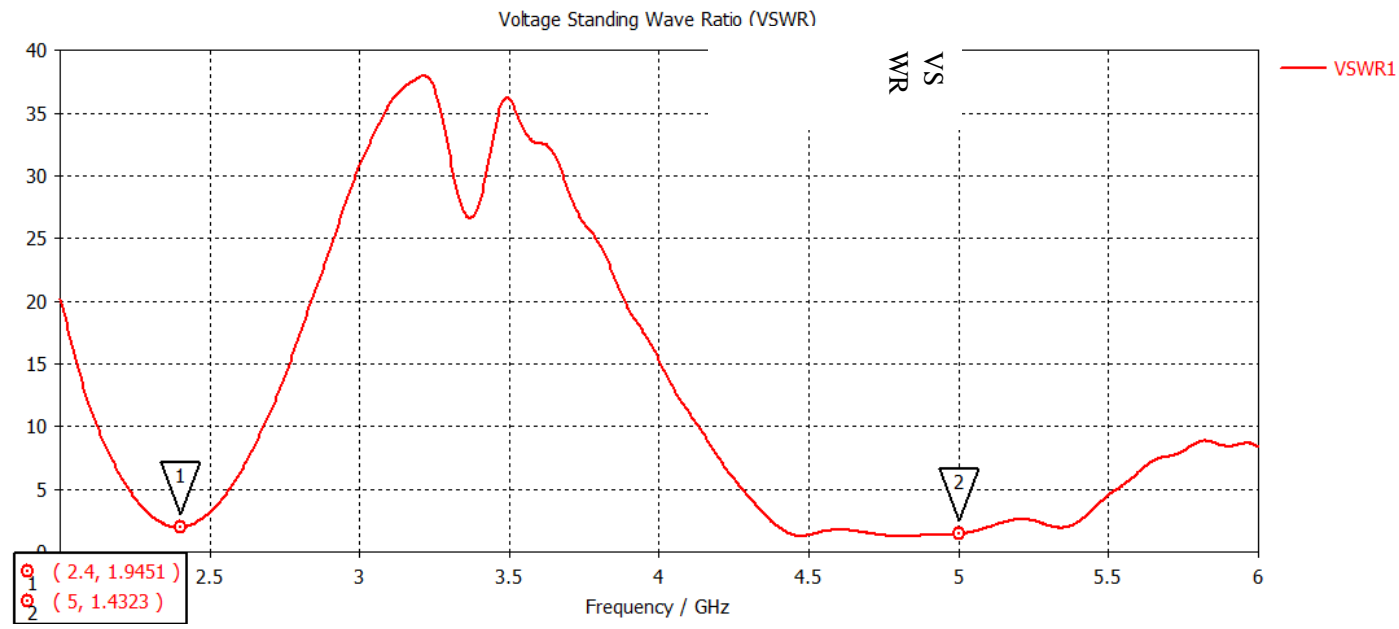

Figure 6 VSWR curve with optimized parameters.

\subsubsection{Gain}

From the antenna simulation the gain value that has been obtain is $3.5 \mathrm{~dB}$. Figure 7 shows the gain value from software simulation
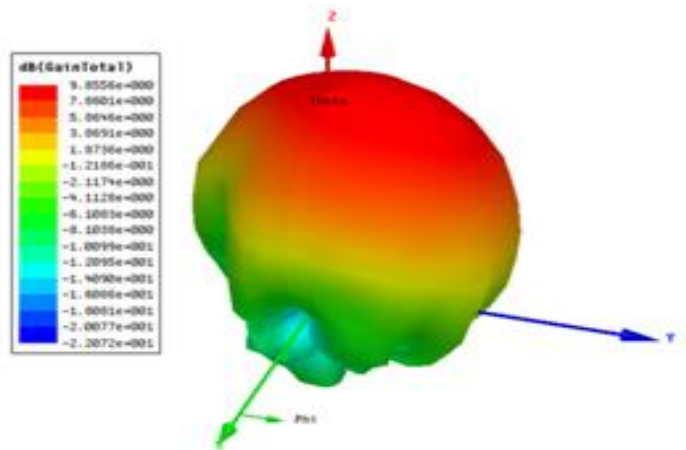

Figure 7 Parameter and Radiation pattern in 3-D

Figure 7 shows the radiation pattern produced by a directional pattern antenna so that at a frequency of 2.4 GHZ it has a non-circular polarity, but an oval which has a large main lobe in front of the patch with a directivity value of $5.474 \mathrm{dBi}$ at a frequency of $2.4 \mathrm{GHz}$. From the antenna parameters generated from the STB device for testing ZigBee technology, the antenna can be implemented on a WSN-based device which is a standard from IEEE 802.15.4. The ZigBee module functions as a wireless link to another device or what is currently commonly referred to as Machine to Machine (M2M). In its implementation, ZigBee can manage its own network, including managing data exchange between machines in its network. Embedded systems (embedded applications) are one example where ZigBee is commonly applied.

\section{Conclusion}

In this paper the writter designed an microstrip antenna with rectangular microstrip antenna Patch Dual Slit Vertical with Ground Square Design using feed with the dimension of $1 \mathrm{~mm} \times 1 \mathrm{~mm}$ and using two rectangular slot on the ground plane has resulting $2.4 \mathrm{GHz}$ bandwidth from the range frequency of $2.4-2.425 \mathrm{GHz}$. The gain value of this antenna simulation is $3.5 \mathrm{~dB}$ with VSWR value not more than 2 that is 1.7. From this simulation result the antenna can be applied in Wireless Personal Area Network application.

\section{ACKNOWLEDGMENTS}

We thank the Ministry of Research, technology and higher education and LPPM Unisma Bekasi for facilitating the research planning until this proposal has been passed.

\section{REFERENCES}

[1] Lim Eng Gee, et al (2010)." Ultra wideband antennas-past and present", IAENG international Journal of Cumouter Science, Dr.S.S.Riaz Ahamed (2005), The Role Of Zigbee Technology In Future Data Communication System, Sathak Institute of Technology

[2] Gislason Drew. Zigbee Wireless Networking. 1st Edition. Paperback ISBN: 9780750685979 . eBook ISBN: 9780080558622. Published: 21st August 2008

[3] Patrick Kinney (2003), ZigBee Technology: Wireless Control that Simply Works, white paper 
[4] Nofianti, Dwi (2011). "Simulasi Kinerja Wpan 802.15.4 (Zigbee) Dengan Algoritma Routing Aodv Dan Dsr. Thesis Magister Ft Undip, Semarang

[5] M. Nasher-Moghadasi, R.Sadeghzadeh, L. Asadpor and B.S. Virdee, A small dual-band CPW-fed monopole antenna for GSM and WLAN applications, IEEE Antennas Wireless Propagation Letters,Vol.12, pp. 508-511,Apr 2013.

[6] Xiao Lei Sun, Li Liu, S.W. Cheung and T. I. Yuk, Dual-band antenna with compact radiator for 2.4/5.2/5.8 GHz WLAN applications, IEEE transactions on Antennas and Propagation, VOL. 60, No. 12, December 2012.

[7] A. Khaleghi, Dual-band Meander line antenna for wireless LAN communication, IEEE Transactions on Antenna Propagation, Vol.55, No. 3, pp. 10041009, Mar 2007

[8] Neda Noorani, "Wireless Multi-Sensor Monitoring System Utilizing IEEE 802.15.4 Communication Standards for Water Leakage Detection", Department of Electrical and Computer Engineering
[9] Y.S. Wang, M.C. Lee and S.J. Chung, Two PIFArelated miniaturized dual-band antennas, IEEE Transaction Antennas and Propagation, Vol. 55, No.3, pp.505-511, March 2007

[10] M.Ravi kishore, A.Janardhana, "Design \& Performance Analysis of Double U-Slot Microstrip Antenna for WiMAX Application" International Journal of Advanced Research in Electronics and Communication Engineering (IJARECE), vol. 3, issue 8, August 2014.

[11] Ojha, John R, Marc Peters, 2010 . Patch Antennas and Microstrip Lines. Publisher InTech.

[12] P. Yang, F. Yang, Z. P. Nie , H. J. Zhou, B. Li, and X. F. Tang "Robust Beam-former Using Manifold Separation Technique for Semispherical Conformal Array" IEEE Antennas And Wireless Propagation Letters, Vol. 10, 20111035

[13] Ramesh Garg, Prakash Bartia, Inder Bahl, Apisak Ittipiboon, 'Microstrip Antenna Design Handbook', 2001, pp 1-68, 253-316 Artech House Inc. Norwood, MA. 